\title{
Design of Large Scale Detectors Based on Polystyrene Solid Scintillators Made of Granulated Polystyrene with WLS Fibers Light Collection
}

\author{
Aleksandr M. Gorin ${ }^{1}$ \\ State Research Center of Russian Federation, Institute for High Energy Physics (IHEP), Research Centre \\ Kurchatov Institute \\ 1 Nauki sq, Protvino, Moscow region, 142281, Russia \\ E-mail: gorineihep.ru

\section{Mikhail V. Medynsky} \\ State Research Center of Russian Federation, Institute for High Energy Physics (IHEP), Research Centre \\ Kurchatov Institute \\ 1 Nauki sq, Protvino, Moscow region, 142281, Russia \\ E-mail: Mikhail.Medynsky@ihep.ru
}

\section{Vera V. Morozova}

State Research Center of Russian Federation, Institute for High Energy Physics (IHEP), Research Centre Kurchatov Institute

1 Nauki sq, Protvino, Moscow region, 142281, Russia

E-mail: mor2vemail.ru

\section{Vladimir I. Rykalin}

State Research Center of Russian Federation, Institute for High Energy Physics (IHEP), Research Centre Kurchatov Institute

1 Nauki sq, Protvino, Moscow region, 142281, Russia

E-mail: Vladimir.Rykalin@ihep.ru

\section{Valeri V. Volkov}

State Research Center of Russian Federation, Institute for High Energy Physics (IHEP), Research Centre Kurchatov Institute

1 Nauki sq, Protvino, Moscow region, 142281, Russia

E-mail: Valeriy.Volkoveihep.ru

\footnotetext{
${ }^{1}$ Speaker

(c) Copyright owned by the author(s) under the terms of the Creative Commons 


\begin{abstract}
Test results of $50 \times 50 \mathrm{~cm}^{2}$ area scintillation detectors, produced of the commercial polystyrene granules with a technique of melting in a polished molding box are presented in the paper. The scintillators maximum emission spectrum is $425 \mathrm{~nm}$ and in comparison with block polymerized scintillators they have the same light yield in small samples, but significantly smaller volume transparency. The efficient scintillation light collection is realized with the help of $1 \mathrm{~mm}$ WLS fibers glued in grooves on the surface of the scintillator. An avalanche multipixel photodiode (SiPM) with $2.5 \times 2.5 \mathrm{MM}^{2}$ sensitive area is used as a photo receiver.

The counter light yield for MIP detection reaches 60 photoelectrons, light collection nonuniformity does not exceed $30 \%$. For example, calculated MIP detection efficiency is $99.9 \%$ at 15 phe discriminator threshold and in this case the peak-value ratio in an amplitude spectrum is equal to 1.2 and event rate is about 100 1/s. The noise counts created by only SiPM are not observed at this threshold.

The small sizes of SiPM and preamplifier allows one to create detectors without geometrical inefficiency zones and to cover with them areas of any size. Such detectors may be used in cosmic ray researches, guard systems and other applications.
\end{abstract}

International Conference on New Photo-detectors

PhotoDet 2015

6-9 July 2015

Moscow, Troitsk, Russia 


\section{Introduction}

Detectors based on polymer scintillators are widely used in high energy physics experiments and other fields of science and engineering. Development and improvement of the detectors is provided by the design of new devices and materials. Thus, recently developed avalanche multipixel photodiodes have got high quantum efficiency and large gain. These diodes obtained the name of the silicon photomultipliers - SiPM. To collect scintillation light wave length shifting (WLS) fibers having a large attenuation length of light and good timings are widely used. The technique of manufacturing bulky scintillators using industrial polystyrene granules (BASF 165H) by melting in the mold was developed at IHEP [1]. The granules are mixed with scintillation dyes, placed into molds with polished surfaces, and melted in a vacuum furnace. Such technology allows one to increase the rate of scintillators production, and currently our equipment allows one to produce up to $10-15$ tons of scintillators a year. Dimensions of the scintillator plates are up to $60 \times 100 \mathrm{~cm}^{2}$ (limited by furnace size), tested thickness - from 20 to $50 \mathrm{~mm}$ and small cross section long (up to $3 \mathrm{~m}$ ) strips are also possible.

In this paper we discussed the possibility of creating scintillation detectors by combining the above elements into a single system. Rather long WLS fibers are placed uniformly on the wide side of the scintillator, and their ends are directed to a small area SiPM.

\section{Detector design}

We have made scintillator plates of $50 \times 50 \mathrm{~cm}^{2}$ size with a thickness of 20 and $50 \mathrm{~mm}$. These scintillators have a maximum of irradiation spectrum of $425 \mathrm{~nm}$, and compared with polymerized from styrene, have similar light output in small samples (of the order of several $\mathrm{cm}$ ), but significantly lower volume transparency. These plates were used for detector prototypes with WLS-fibers light collection. $1 \mathrm{~mm}$ diameter WLS fibers (Kuraray, Japan) Y-11 (200 ppm) type [2] with a maximum of emission spectrum of the $476 \mathrm{~nm}$ (in zero length of the fiber; after $1 \mathrm{~m}$ - about $500 \mathrm{~nm}$ ) and not less than $3.5 \mathrm{~m}$ attenuation length. Two fibers are glued in the spiral grooves $1.5 \mathrm{~mm}$ wide and $2 \mathrm{~mm}$ deep, machined on the surface of the scintillator. The ends of the fibers ( 4 pcs.) are attached to the scintillator corner, fixed in a square $2 \times 2 \mathrm{~mm}^{2}$ in size and carefully polished. In the first counter (further version 1 ) the total length of the fibers is about $6 \mathrm{~m}$, and in the second (further version 2) is about $4 \mathrm{~m}$ (economical version). A sketch and a photo of the fibers (shown in green) arrangement in the first counter is shown in Fig.1. The idea of this solution comes from [3].
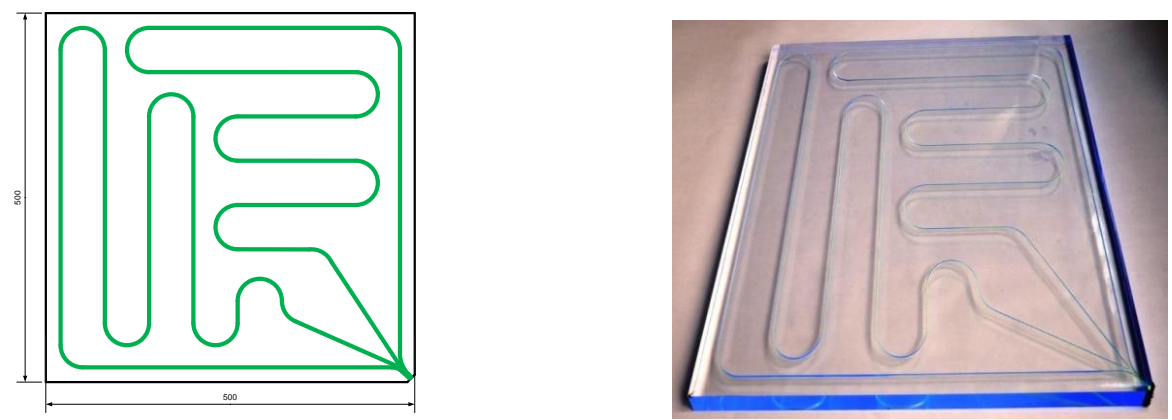

Fig.1. Scintillator plates. The arrangement of fibers. The bending radius of the fibers is $30 \mathrm{~mm}$. 


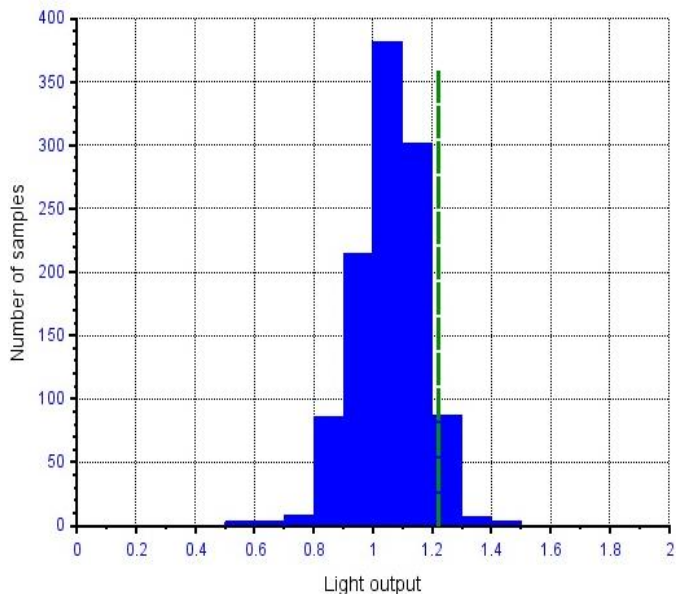

We produced more than 1200 of such plates $50 \times 50 \mathrm{~cm}^{2}$ in size for Baksan Observatory. Histogram of the light output of these scintillators tested with $\gamma$-source is shown in Fig.2.

The counter with $5 \mathrm{~cm}$ thick scintillator with the geometry of version 1 was fabricated. The scintillators are wrapped in one layer of $30 \mu \mathrm{m}$ aluminized Mylar, and then black/ red paper (100 $\mu \mathrm{m})$ for the light tightness. A large area detector may be easily combined with these plates having gaps in between less than $0.5-1 \mathrm{~mm}$, which is important for guard systems.

Fig.2. Relative light output of $\sim 1100$ pes of $5 \mathrm{~cm}$ scintillation plates.

Green dash line - Kharkov ordinary PS scintillator.

The same SiPM of CPTA 150-50 type [4] with a size of the sensitive area of $2.5 \times 2.5$ $\mathrm{mm}^{2}$ was used as a photo sensor. This SiPM has $600 \mathrm{~nm}$ maximum spectral sensitivity, a gain up to $10^{6}$, capacitance $200 \mathrm{pF}$ at an operating voltage of $42-44 \mathrm{~V}$. To fix a photodetector, the angle of the scintillator plate is cut so as to obtain the pad size $10 \times 15 \mathrm{~mm}^{2}$. SiPM was carefully aligned relative to output ends of WLS-fibers and fixed on the corner of the scintillator plate without the use of optical compound. The negative bias voltage was applied to the photodiode.

An electric SiPM signal is fed to a small amplifier with an

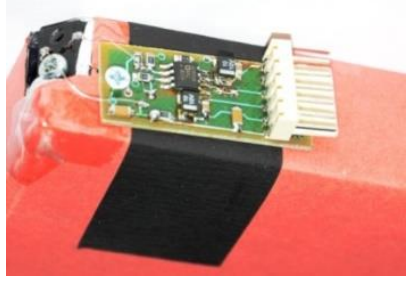
input impedance of $50 \mathrm{Ohms}$, gain of 10 and the rise time of about $3.5 \mathrm{~ns}$. The amplifier is located on the scintillator directly near the photodiode and generates a signal into a coaxial cable with 50 Ohm impedance, as shown in Fig.3.

Fig.3. Positioning of the SiPM (left) and preamplifier (right) on the corner of scintillator.

\section{Experimental results}

ADC scale has been calibrated (see Fig.4) before the measurements. A blue LED low illumination was used in this test, and the distance between the peaks of the separated photoelectrons (pedestal -1 phe -2 phe -3 phe) was measured. Under the assumption of the SiPM linearity the average value of the single-electron peak (SER) is $13.1 \mathrm{ADC}$ channels with an operating voltage $-43.5 \mathrm{~V}$, and this voltage and SER were used in other measurements.

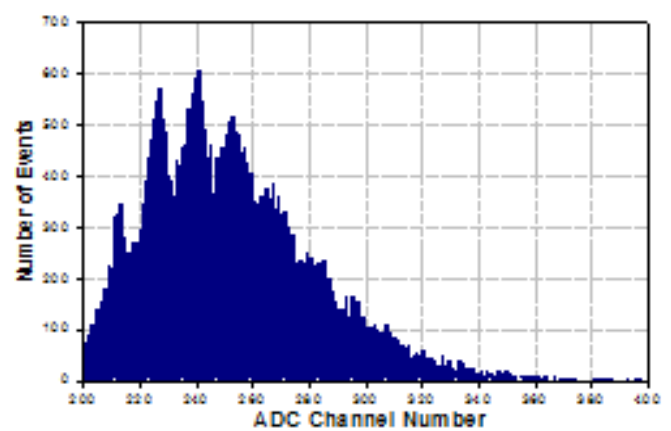

Fig.4. SiPM amplitude spectrum. Left peak is pedestal. 
The measurements were performed with cosmic muons using two $10 \times 10 \mathrm{~cm} 2$ size trigger counters, located above and below the scintillator plate. Amplitude spectra were collected with a 12-bit ADC (from GAMS experiment) with gate duration of $50 \mathrm{~ns}$. An example of the amplitude spectrum for $20 \mathrm{~mm}$ scintillator counter with a modest light output (version 2) is shown below - Fig.5. Dependence of the detection efficiency calculated from the amplitude spectrum as a function of the threshold (ADC channel units) is shown in Fig. 6.

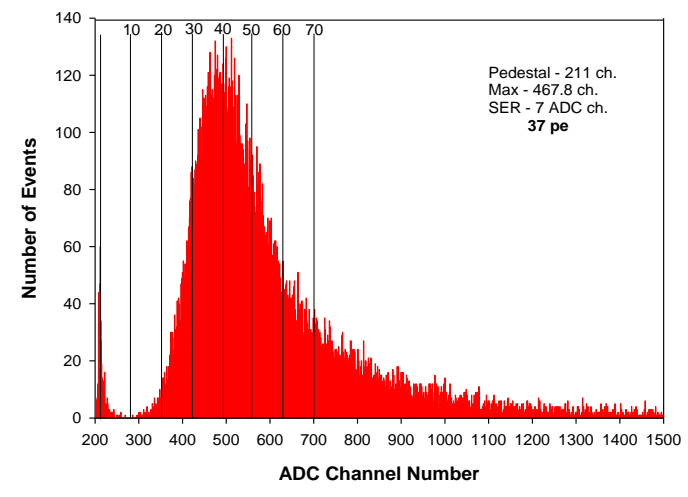

Fig.5. Counter version 2. Trigger counters are in the centre of plate. Labeled black

lines - number of photoelectrons.

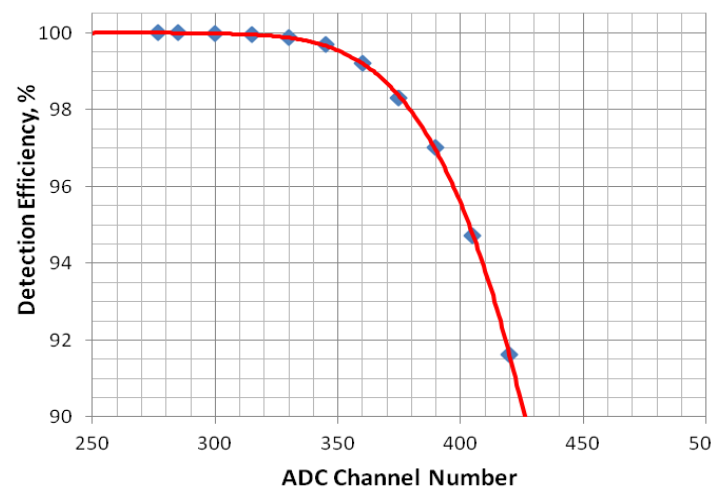

Fig.6 Detection Efficiency calculated from the amplitude spectrum data.

DE at 320 ADC ch. is $99.9 \%$

The results of the measurements are shown in Table 1. The left edge of the amplitude spectrum was fit with a normal distribution ("Amplitude"). Light collection nonuniformity does not exceed $30 \%$. The noise of SiPM avalanche multiplication may be completely suppressed at $\sim 20$ phe detection threshold.

Table 1. Detectors light output. Cosmic ray test with external trigger $\left(10 \times 10 \mathrm{~cm}^{2}\right)$

\begin{tabular}{|c|c|c|c|}
\hline $\begin{array}{l}\text { Detector version } \\
\text { Thickness, mm } \\
\text { SER, ADC ch. }\end{array}$ & $\begin{array}{c}\text { Trigger coun- } \\
\text { ter } \\
\text { position }\end{array}$ & $\begin{array}{l}\text { Amplitude, } \\
\text { ADC channels }\end{array}$ & $\begin{array}{c}\text { Number } \\
\text { of photoelectrons }\end{array}$ \\
\hline \multirow{3}{*}{$\begin{array}{c}1 \\
20 \\
13.1\end{array}$} & Centre & 762.8 & 58 \\
\hline & Far corner & 780.9 & 60 \\
\hline & Close corner & 925.3 & 71 \\
\hline \multirow{2}{*}{$\begin{array}{c}2 \\
20 \\
13.1\end{array}$} & Centre & 541.2 & 41 \\
\hline & Far corner & 566.3 & 43 \\
\hline \multirow{3}{*}{$\begin{array}{c}1 \\
50 \\
38.1\end{array}$} & Centre & 1074.6 & 28.2 \\
\hline & Far corner & 893.6 & 23.5 \\
\hline & Close corner & 1243.1 & 32.7 \\
\hline
\end{tabular}

The use of such counters may be interesting for cosmic ray researches, where large detector areas are required. In this case the counter can operate with self-trigger with a properly chosen threshold. To check such mode of operation the counter with lower light output (version 


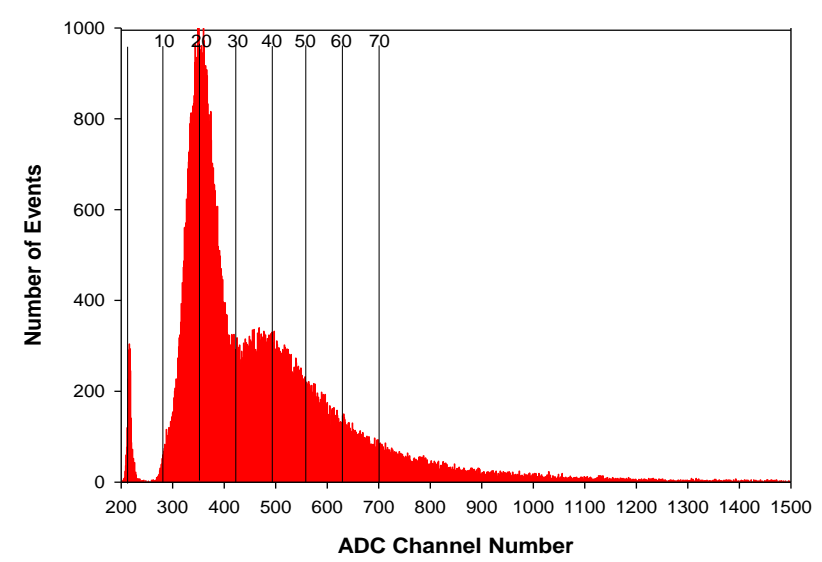

$2,-2 \mathrm{~cm}$ thick scintillator) was tested. The output signal of the amplifier is split using a resistive divider and fed to the $\mathrm{ADC}$ and discriminator. The threshold of the discriminator was set at 15 photoelectrons $(20 \mathrm{mV} \leftrightarrow 105 \mathrm{ADC}$ ch.) with the maximum of the cosmic muons spectrum of 40 phe. The obtained result is shown in Fig.7. The position of spectral maximum is in a good agreement with the result obtained with an external trigger (see Fig.5).

Fig.7. Self-triggering counter version 2 amplitude spectrum. Detection Efficiency - 99.9\%, peak/value ratio - 1.18.Pedestal $-214.5 \mathrm{ch}$. was accumulated separately (random trigger).

An external oscillator $(1000 \mathrm{~Hz})$ supplies random trigger signal which allows us to estimate fraction of random coincidence at high statistics (order of $10^{6}$ ):

- $1.6 \times 10^{-5}$ at 15 photoelectron threshold (signals above $325 \mathrm{ADC}$ ch.),

- $1.0 \times 10^{-5}$ at 17 photoelectron threshold (signals above 339 ADC ch.).

For the insulated SiPM (disconnected from the detector and light isolated) we have got the following random coincidence:

- $7.3 \times 10^{-6}$ at 15 photoelectron threshold,

- $2.4 \times 10^{-6}$ at 17 photoelectron threshold.

Thus, the number of random coincidences at high detection efficiency (above 99\%) seems to be reasonably low.

\section{Muon Detector}

The same technology was developed for the production of the long thin strips which are widely used for muon detectors.

The sketch of the muon hodoscope element (strip) is shown in Fig.8. Thickness of the scintillator is $10 \mathrm{~mm}$. Two $1 \mathrm{~mm}$ fibers (Y11) 4 m long are glued with epoxy into the grooves of $1.5 \times 2 \mathrm{~mm}^{2}$ cross-section. The element is wrapped with Tyvek $(100 \mu \mathrm{m})$ and black/red paper $(100 \mu \mathrm{m})$ for the light insulation. The muon detector is planned to cover large area as $2 \times 2 \mathrm{~m}^{2}$ in OKA experiment at IHEP.

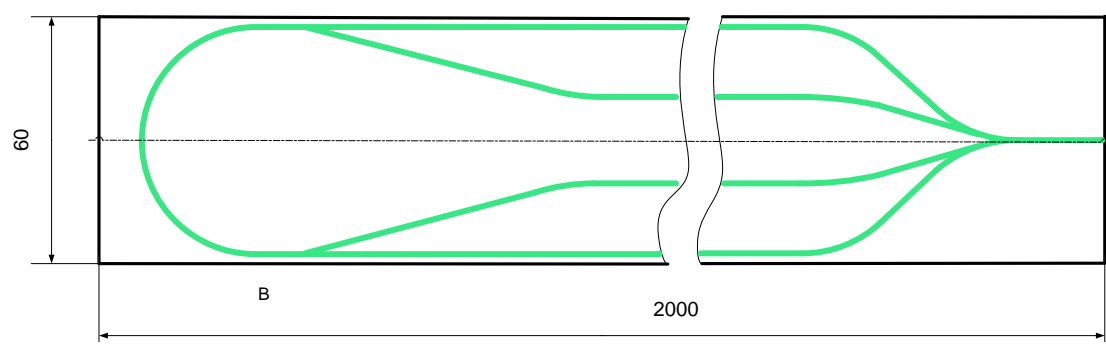

Fig.8. The sketch of the muon strip. WLS fibers are shown in green. 
Typical test results are shown in Fig.9 (cosmic ray test with external trigger) and Fig.10 (OKA beam test).

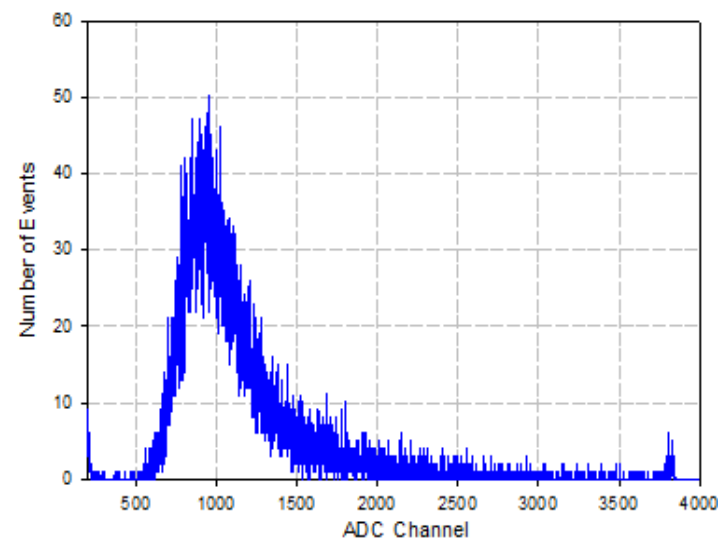

Fig.9. Cosmic ray amplitude spectrum (SiPM No9@ 43,8 V): distance - 150 cm, 45 pe L.Y. Light attenuation along the strip $(\mathrm{max} / \mathrm{min})$ not exceeding $30 \%$

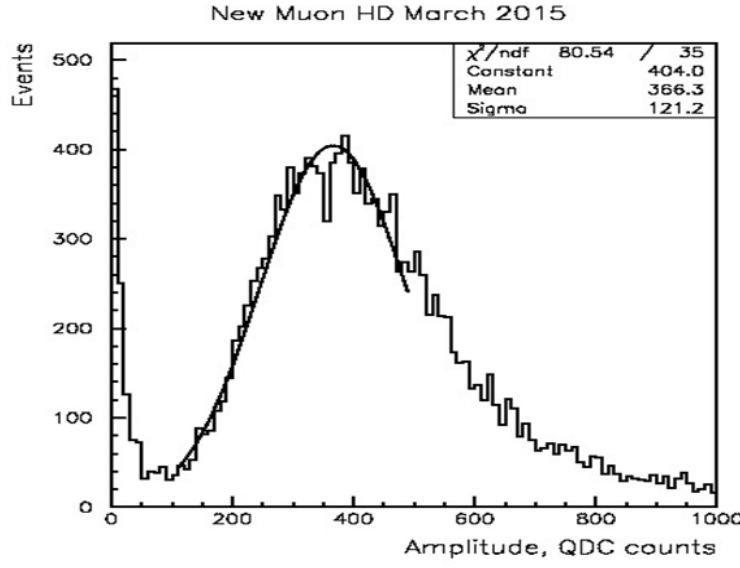

Fig.10. Muon amplitude spectrum obtained at OKA beam test: distance $-100 \mathrm{~cm}$. Measured light fluctuation along the strip not exceeding $\pm \mathbf{5 \%}$

The strips may be produced up to $3 \mathrm{~m}$ long, with a total volume of $~ 1000$ strips per year.

\section{Conclusion}

The aim of this work is the development of a simple and robust design of the detector which does not have geometric dead zones. The tests of the counters, made on the basis of the solid scintillators of polystyrene granules with the WLS fiber light collection and readout with SiPM, show that:

1. The use of such scintillators is very promising. The light output above 30 photoelectrons ensures high detection efficiency. It may be improved with better quality SiPM selection or use of another type avalanche photodiodes.

2. The use of long WLS fibers enables effective light collection with small nonuniformity from the large area of scintillator to a small area of SiPM;

3. Small size SiPM and preamplifier results in a lack of geometrical dead zones of the detector;

4. The detector has got simple and reliable design.

This work was performed under the Contract number N.4h.44.90.13.1118 from 31.05.2013.

\section{References}

[1] G.I. Britvich, V.V. Brekhovskikh, V.K. Semenov, S.A. Kholodenko, "The Main Characteristics of Polystyrene Scintillators Produced at IHEP”, Preprint IHEP 2013-23 (in Russian).

[2] http://kuraraypsf.jp/psf/ws.html

[3] Yu.V. Musienko, et al., "High Sensitive Micropixel Avalanche Photodiodes for Scintillation Counters of the Near Detector of T2K experiment", PTE, 2008. No 51. pp. 101-107.

[4] http://www.cpta-apd.ru/PDF/CPTA\%20MRS-APD\%20avalanche\%20photodiode\%20ru15050.pdf 